\title{
A 4-METER McMATH TELESCOPE FOR THE INFRARED
}

\author{
W. LIVINGSTON \\ National Solar Observatory, P. O. Box 26732, Tucson, AZ 85726, U.S.A.
}

\begin{abstract}
Having no window and a filled aperture, i.e., no occlusion by secondary optics, the all-reflective McMath telescope is a proven IR facility. Beyond about $2 \mu \mathrm{m}$, it is diffraction limited, however. Engineering studies show that the McMath building could accommodate an increase to a 4-m aperture with a 6-m alt-azimuth feed, permitting sub-arcsec resolution to $12 \mu \mathrm{m}$. The use of cooled, solid aluminum mirrors would eliminate "mirror seeing", which plagues non-vacuum solar telescopes.
\end{abstract}

Key words: infrared: stars - Sun: general - telescopes

\section{Introduction}

\subsection{Why Observe the SUn in the Infrared?}

As well discussed in this symposium, the IR offers many advantages for observational study of the Sun. For example, at $1.6 \mu \mathrm{m}$ the photospheric opacity reaches a minimum, and we see $\sim 40 \mathrm{~km}$ deeper there, allowing the design and verification of convection models. Away from telluric absorption bands there is a true infrared continuum, as opposed to visible wavelengths, where line-blanketing is ubiquitous. Continuum opacity beyond $1.5 \mu \mathrm{m}$ is almost entirely due to $\mathrm{H}^{-}$free-free absorption, which is well understood.

Molecular bands of $\mathrm{CO}$ around 2.3 and $4.7 \mu \mathrm{m}$ are excellent thermal probes of the temperature minimum and, at high spatial resolution, should permit the study of apparent thermal bifurcations in the outer layers that puzzle us.

Most exciting of all is the possibility of mapping the full vector form of surface magnetism. Zeeman splitting increases as $\lambda^{2}$, while Doppler broadening increases only as $\lambda$. In the visible, plage field strengths must be inferred by modeling. The $g=3$ line of Fe I $1.5648 \mu \mathrm{m}$ is completely split in most plage regions allowing the direct measurement of field strength. Flux tubes of 1-2 kG are the rule, but there remains the question of the role of weak fields, how fields evolve, and the possible existence of a "turbulent" component. At $12.5 \mu \mathrm{m}$, telescope polarization becomes negligible and vector magnetic fields can be directly deduced, see Harvey (1985). Table I summarizes the various wavelengths and spectral lines now of interest in the infrared.

\subsection{Infrared Facility Requirements}

What are the desired features for a solar infrared telescope? Assuming the observational bandwidth to be 0.3 to $12.5 \mu \mathrm{m}$, we come up with the following needs, roughly in order of priority:

1. All reflecting system: For reasons of achromatic transmission, no windows are allowable. This rules out a vacuum-telescope configuration.

2. Aperture $\geq 4$ meters: The diffraction limit, in arcsec, is given by $\Delta \theta \approx 0.25 \lambda / D$, where $\lambda$ is in microns and $\mathrm{D}$ is the aperture, in meters. The present $1.5-\mathrm{m}$ 
TABLE I

Infrared Diagnostics of the Solar Atmosphere.

\begin{tabular}{|c|c|c|c|c|c|c|}
\hline ID & $\lambda(\mu \mathrm{m})$ & $g$ & $\bar{U}$ & $\mathrm{P}$ & Application & References \\
\hline $\mathrm{FeH}$ & 1.006319 & 0 & $\bar{x}$ & & umbrae, pores & 1 \\
\hline $\mathrm{FeH}$ & 1.006270 & $\sim 1$ & $\mathbf{x}$ & & umbrae, pores, mag. field & \\
\hline $\mathrm{He}$ & 1.0830 & 1.2 & & & high chromosphere & \\
\hline $\mathrm{H}(\mathrm{P} \beta)$ & 1.2818 & $\sim 1$ & & & chromosphere & 2 \\
\hline $\mathrm{Fe}$ & 1.564852 & 3 & $\mathbf{x}$ & $\mathbf{x}$ & plage, umbrae mag. field & $3,4,5,6$ \\
\hline cont & 1.63 & - & $\mathbf{x}$ & $\mathbf{x}$ & opacity minimum & 7 \\
\hline $\mathrm{Na}$ & 2.208367 & 1.3 & $\mathbf{x}$ & $\mathbf{x}$ & no $\pi$-component & 8 \\
\hline $\mathrm{Ti}$ & 2.227358 & 2.5 & $\mathbf{x}$ & & umbrae, pore mag field & 8 \\
\hline $\mathrm{CO}$ & 2.324 & - & $\mathbf{x}$ & $\mathbf{x}$ & $\mathrm{dT} / \mathrm{dh}$ in cool parts & 9 \\
\hline $\mathrm{H}$ & 4.0512 & - & - & - & prominences, Stark effect & 10 \\
\hline $\mathrm{CO}$ & 4.665 & - & $\mathbf{x}$ & $\mathbf{x}$ & oscillations, $\mathrm{dT} / \mathrm{dh}$ & 11 \\
\hline cont & 10.0 & - & $\mathbf{x}$ & $\mathbf{x}$ & limb dark, spots, seeing & $12,13,14$ \\
\hline $\mathrm{He}$ & 10.879 & - & - & - & prominences & 15 \\
\hline $\mathrm{OH}$ & 11.065 & - & & $\mathbf{x}$ & chromosphere oscillations & 16 \\
\hline $\mathrm{H}$ & 11.306 & - & - & - & prominences & 15 \\
\hline $\mathrm{Mg}$ & 12.320 & 1.0 & & $\mathrm{x}$ & high photosphere mag. field & 17,18 \\
\hline
\end{tabular}

where $g=$ Landé factor, $\mathrm{U}=$ umbra, $\mathrm{P}=$ photosphere, $\mathrm{dT} / \mathrm{dh}$ implies thermal structure. References:

1 Wallace 1991; 2 Livingston 1990; 3 Harvey and Hall 1975; 4 Solanki et al. 1990; 5 Stenflo et al. 1987; 6 Rabin et al. 1990; 7 Foukal et al. 1990; 8 Hall 1970; 9 Ayres and Testerman 1981; 10 Foukal and Hinata 1991; 11 Ayres and Brault 1990; 12 Boyd 1978a; 13 Kopp and Livingston 1991; 14 Turon and Lena 1970; 15 Zirker 1985; 16 Deming et al. 1984; 17 Deminget al. 1991; 18 Carlsson et al. 1992.

McMath, thus, has a diffraction limit of $2.1^{\prime \prime}$ at $12.5 \mu \mathrm{m}$; a 4-m McMath would have $0.8^{\prime \prime}$.

3. Filled aperture: In the thermal IR a cold aperture stop in the cryogenic detector should preferably see no telescope structure, ruling out Cassegrain optics.

4. Long focal length: A short-focus 4-m telescope becomes, in effect, a solar furnace. Even if the temperature of secondary mirrors could be adequately cooled, the radiation flux of an $f / 1$ beam will cause heating of the air near focus with resulting turbulence and uncorrectable induced seeing. Filters will break, and other damage will be expected. The solution is a long-focal-length instrument which avoids any major concentration of heat.

5. Adaptive optics: Atmospheric seeing correction is much easier in the IR than visible. Refer to Table 2 in Roddier and Graves (1993), in these proceedings. At $\lambda=0.5 \mu \mathrm{m}$, to obtain the optimum Strehl ratio of 0.3 requires 90 compensating Zernike modes for a field of view (isoplanatic patch) of $2.6^{\prime \prime}$. At $\lambda=2.2 \mu \mathrm{m}$, the same compensation is obtained with 2 modes (a simple tip-tilt correction) and the field of view realized is $60^{\prime \prime}$. Roddier and Graves propose a wavefront sensor that works on out-of-focus granulation images. This is untested, however. 
6. Dry site: Certain IR wavelengths are heavily absorbed by water vapor. By locating the facility on a high-elevation dry site, water vapor interference is minimized.

\subsection{Strategy for Creating an IR Facility: a 4-M McMath}

Why the McMath? The answer is, "because it is there." The existing McMath telescope on Kitt Peak already satisfies most of the demands for an IR solar facility: It is all reflective, has a filled aperture, a long focus throw, and plenty of room to install adaptive control. The site is relatively dry except in summer. The main limitation is its small, 1.5-m aperture. Fortunately, owing to conservative design, the building superstructure could easily accommodate an upgrade to a 4-m concave within the tunnel. A 6-m alt-azimuth flat as a light feed would replace the present 2-m heliostat. We believe this proposed upgrade to be especially cost-effective, providing a state-of-the-art telescope for a fraction of the cost of starting from scratch.

\section{Upgrade of the McMath to 4 meters}

Larry Barr has conducted an engineering study that indicates that it is feasible to modify the present structure to accommodate a $6-\mathrm{m}$ feed, a $4-\mathrm{m}$ concave and to provide mirror handling with existing cranes for periodic re-aluminizing. One unavoidable change would be an enlargement of the aluminizing room and the installation of a $6-\mathrm{m}$ coating chamber.

\subsection{The 6-M Light Feed}

Especially challenging is this large assembly, Figure 1 . Barr proposes that the 6-m flat be of solid aluminum, $0.15 \mathrm{~m}$ thick. Discussion with a potential vendor - Rozelot and Leblanc (1991) - indicates such a blank would be cast as subsections, which are then forged, electron-beam welded together, heat-treated by repeated cycling from cryogenic to $+100 \mathrm{C}$ temperatures, surfaced and ground, coated on both sides with a $150 \mu \mathrm{m}$ nickel-sulfate amorphous layer capable of taking a polish, and then figured flat to $\pm 0.5 \mu \mathrm{m}$ overall. Small-scale irregularity would be $\pm 0.1 \mu \mathrm{m}$. The aim here is about $0.5^{\prime \prime}$ resolution in the visible, comparable to what the telescope achieves today.

The mirror would be supported by an array of hydraulic inter-connected assemblies. These push against its back surface in a cell under partial vacuum whose pressure depends on mirror attitude. Thus, the supporting force against the mirror is constant (except for any wind-buffeting component). Number and spacing of the arms has been optimized by computer modeling.

Liquid cooling would be applied to the back of the mirror so as to keep the front surface $<0.5 \mathrm{C}$ from ambient. Even so, a front-to-rear temperature gradient will tend to induce a curvature. This will be countered by the hydraulic arms. The latter also correct for gravity.

We believe this use of a cooled metallic (aluminum) mirror, as opposed to an essentially insulating ceramic (glass) blank, may be of great benefit for non-vacuum 


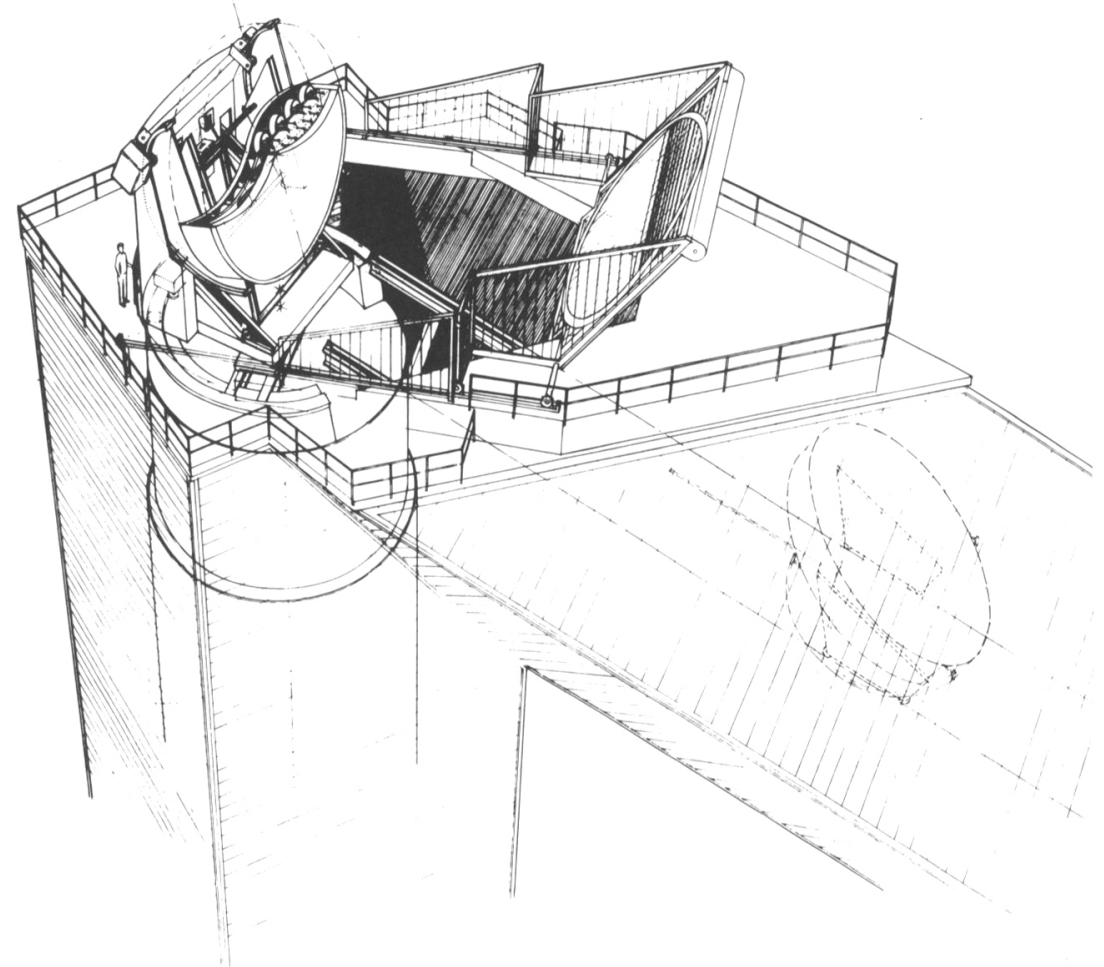

Fig. 1. Rendering of the top of the McMath and the 6-m alt-azimuth flat. Cutaway reveals some of the hydraulic support arms and the cooling channels. Wind fence is shown in its extended position away from the mirror.

solar telescopes. Within seconds after sunlight is incident on a $10 \%$ absorbing aluminum reflecting layer, that layer will rise in temperature if the blank is glass. A 1-C rise initiates "mirror seeing" (Barr et al. 1990; Iye et al. 1991). Experiments to document these thermal optical improvements are planned for this fall (1992).

The mirror and support cell, together with its bearing journals, will be about $30,000 \mathrm{~kg}$. This moving component will be supported by hydrostatic pads in an altazimuth mount. For re-aluminizing, this moving component disengages from the main alt-azimuth column and is lowered down the shaft on self-contained wheels. All sky coverage to a declination limit of $+50^{\circ}$ (with $60 \%$ full aperture) is achieved. Thus, the McMath will become an important 4-m telescope for solar-stellar nighttime use (see Livingston and Barr 1992).

Wind and weather protection are important concerns. Our present wind-fence 
in front of the heliostat has proved remarkably effective in preventing image shake. Before the fence was installed, image motion was noticeable at about $6.5 \mathrm{~m} / \mathrm{s}$; at $18 \mathrm{~m} / \mathrm{s}$ (not uncommon) the heliostat preload was inadequate to prevent tooth-totooth floating at the drive gear. With the wind-fence erected, image motion at 22 $\mathrm{m} / \mathrm{s}$ is comparable to that at $6.5 \mathrm{~m} / \mathrm{s}$ without it, at least for the most common condition of a south wind. For the 6-m, we plan an even more elaborate wind fence which can be moved as close to the mirror as the field of view permits. A badweather and closure mode would involve translating the fence, and rotating the mirror, to bring them into actual sealed contact (through a gasket).

\subsection{The 4-M Concave}

Here we envisage a 4-m meniscus similar to the $6-\mathrm{m}$, i.e., of solid aluminum. It will be likewise cooled and actively supported, although its static orientation leads to certain simplifications. Like the present $1.5-\mathrm{m}$ concave, it will have a focal length of approximately $86 \mathrm{~m}$. An iris diaphragm will probably be used to stop down the mirror for certain visible-light applications.

Figuring a 4-m mirror of such a long focal length presents difficulties to most optical shops. We propose to do the final polishing in situ at the telescope. Testing would be against the present 2-m heliostat at the center-of-curvature.

\subsection{The 1.8-M Flat}

This mirror, which serves to direct the beam to one of several observing stations, is also of solid aluminum. It must be cooled and stressed to induce a slight cylindrical form in order to provide correction for astigmatism introduced by thermal curvature.

Of course, near the focus there will be further optical stops, all cooled. The unvignetted field on the solar image would be $6^{\prime}$.

\section{Project Status}

The feasibility study is now complete. A preliminary cost estimate has been prepared. Barr continues his work on the optimization of mirror-support schemes. As mentioned, we are now preparing for an experiment, using a surplus $127-\mathrm{cm}$ aluminum "RCT" mirror, to measure the influence of temperature control on mirror seeing. Point source images will be recorded with and without incident solar radiation on the mirror and with and without temperature adjustment to ambient.

\section{Acknowledgements}

Larry Barr and the staff of NOAO Engineering and Technical Services are the source of most of the above concepts. Support and encouragement came from Sidney Wolff and John Leibacher. 


\section{References}

Ayres, T. R. and Brault, J. W.: 1990, Astrophys. J. 363, 705.

Ayres, T. R. and Testerman, L.: 1981, Astrophys. J. 245, 1124.

Barr, L., Fox, J., Poczulp, G., and Roddier, C.: 1990, SPIE Proc. 1236, p. 492.

Boyd, R. W.: 1978, J. Opt. Soc. Am. 68, 877.

Carlsson, M., Rutten, R. J., and Shchukina, N. G.: 1992, Astron. Astrophys. 253, 567.

Deming, D., Hewagama, T., Jennings, D. E., and Wiedemann, G.: 1991, in L. November (ed.), Solar Polarimetry, Proc. 11th Sacramento Peak Workshop, NSO, Sunspot, New Mexico, p. 341.

Deming, D., Hillman, J. J., Kostiuk, T., Mumma, M. J. and Zipoy, D. M.: 1984, Solar Phys. 94, 57.

Foukal, P., Little, R., Graves, J., Rabin, D., and Lynch, D.: 1990, Astrophys. J. 353, 712.

Hall, D. N. B.: 1970, Doctoral Dissertation, Harvard Univ., KPNO Contr. No. 556.

Harvey, J. and Hall, D. N. B.: 1975, Bull. Amer. Astron. Soc. 7, 459.

Harvey, J.: 1985, in M. J. Hagyard (ed.) Measurements of Solar Vector Magnetic Fields, NASA Conf. Pub. 2374, NASA, Washington, D.C., 109.

Iye, M., Noguchi, T., Torii, Y., Mikami, Y., and Ando, H.: 1991, Pub. Astron. Soc. Pacific 103, 712.

Kopp, G. and Livingston, W.: 1991, private communication.

Livingston, W. and Barr, L.: 1992 in M. S. Giampapa and J. A. Bookbinder (eds.), Cool Stars, Stellar Systems, and the Sun, Seventh Cambridge Workshop, Astron. Soc. Pacific Conf. Ser. Vol. 26, San Francisco, p. 604.

Rabin, D., Jaksha, D., Plymate, C., Wagner, J., and Iwata, K.: 1991 in L. November (ed.), Solar Polarimetry, Proc. 11th Sacramento Peak Workshop, NSO, Sunspot, New Mexico, p. 361.

Roddier, F. and Graves, J. E.: 1993, these proceedings.

Rozelot, J. P. and Leblanc, J.-M.: 1991, SPIE Proc., 1994, p. 481.

Solanki, S., Biémont, E., and Mürset, U.: 1990, Astron. Astrophys. Suppl. 83, 307.

Stenflo, J. O., Solanki, S. K., and Harvey, J. W.: 1987, A strophys. J. 173, 167.

Turon, P. J. and Lena, P. J.: 1970, Solar Phys. 14, 112.

Wallace, L.: 1991, private communication.

Zirker, J. B.: 1985, Solar Phys. 102, 33-40. 